\title{
Holographic Microwave Imaging Array for Brain Stroke Detection
}

\author{
Lulu Wang', A. M. Al-Jumaily ${ }^{1}$, Ray Simpkin ${ }^{2}$ \\ ${ }^{1}$ Institute of Biomedical Technologies, Auckland University of Technology, Auckland, New Zealand; ${ }^{2}$ Callaghan Innovation, Auck- \\ land, New Zealand \\ Email: luwang@aut.ac.nz, ahmed.al-jumaily@aut.ac.nzm,ray.simpkin@callaghaninnovation.govt.nz
}

Received May, 2013.

\begin{abstract}
This paper proposes a new holographic microwave imaging array (HMIA) technique for brain stroke detection. This approach is based on holographic microwave and aperture synthesis imaging techniques. The system is designed for operation at a single frequency of $2.5 \mathrm{GHz}$. A realistic three dimensional (3D) head model that contains skin, fat, skull, cerebrospinal fluid (CSF), grey matter, white matter and ischemic or hemorrhagic stroke area is developed using MATLAB to demonstrate the proposed HMIA imaging algorithm. A matching solution medium is used between the antennas and the head model. The study is conducted using HMIA computer simulations and 3D head model withstroke. The simulation results showed that small stroke area $(5 \mathrm{~mm}$ in diameter) could be successfully detected with the HMIA approach.
\end{abstract}

Keywords: Microwave Imaging; Holographic Microwave Imaging Array; Aperture Synthesis Imaging; Brain Imaging; Brain Stroke

\section{Introduction}

A brain stroke is the third leading cause of death after the heart disease and cancer [1]. There are two types of brain stroke, ischemic and hemorrhagic. Ischemic stroke accounts for approximately $87 \%$ of all strokes. Acute is chemic strokes occur as a result of an obstruction within a blood vessel supplying blood to the brain. Hemorrhagic strokes result from bleeding within the brain or in the space surrounding. Both medical conditions lead to death in the intermediate future if left untreated. Moreover, the symptoms can be similar between the two conditions; however the medical treatment is significantly different. An incorrect determination of the stroke can lead to the death of the patient. The risk factors include old age, hypertension, or transient ischemic attack, diabetes, high cholesterol, cigarette smoking, and atrial fibrillation [2].

The current clinical imagingdiagnosis tools for stroke detection include Computed Tomography (CT), Magnetic resonant Imaging scanning (MRI), Positron Emission Tomography (PET) and ultrasound [3].The main clinical imaging tools for brain stroke detection are $\mathrm{CT}$ and MRI. Unfortunately these tools are not suitable for continuous monitoring of a stroke's evolution due to cost, time consuming imaging operations and the imaging equipment is not portable. Moreover, CT imaging uses ionizing radiation that is harmful to the patient. These circumstances motivate the interest for new technologies that can integrate with currently available imagining technologies to improve the overall effectiveness of the diagnosis [4].

Microwave based imaging techniques create a map of electromagnetic wave scattering arising from the contrast in the dielectric properties of different tissues and have been investigated as one of the most promising medical imaging tools for many years [5-6]. The advantages of microwave imaging include a whole view of body tissue, lower cost, more comfortable and no radiations. Recent investigations [7-9] indicated that microwave imaginghas the potential to determine perfusion related changes in the human brain and microwave based imaging approaches could be developed as a useful new imaging modality for stroke management.

Unlike these groups [7-9], we propose a new Holographic Microwave Imaging Array (HMIA) technique for stroke detection, which is based on the aperture synthesis far-field imaging technique. Recently, HMIA technique for breast cancer detection has been proposed [10-11]. HMIA of the brain presents a significant challenge, as the brain is an object of interest that is located inside a high dielectric contrast shield, comprising the skull (with low dielectric contrast $\varepsilon=10 \sim 15$ ) and cerebral spinal fluid (with high dielectric contrast $\varepsilon=55 \sim 60$ ) 
[7]. The aim of this study is to assess the feasibility and potential performance characteristics of the HMIA system for brain stroke detection.

\section{Theory}

Figure 1 shows the block diagram of the HMIA system. The system contains an array of 16 small antennas, one is the transmitter and others are receivers, which are located around the head model in far-filed distance. The space between the head model and antenna array is filled with the matching solution medium. The system is designed for operating at a single frequency of $2.5 \mathrm{GHz}$ [7]. When the transmitter transmits the electromagnetic field, the other15 antennas receive the scattered electromagnetic field. The HMIA measures the scattered radiation of the head, which is composed of a set of correlation interferometer pairs. The electromagnetic signals received by each pair are cross-correlated to get the visibility function. A head scattering intensity distribution is formed by applying an inverse Fourier transform to the complex visibility data. Then a $2 \mathrm{D}$ projection image on a $2 \mathrm{D}$ plane of a $3 \mathrm{D}$ head is generated.

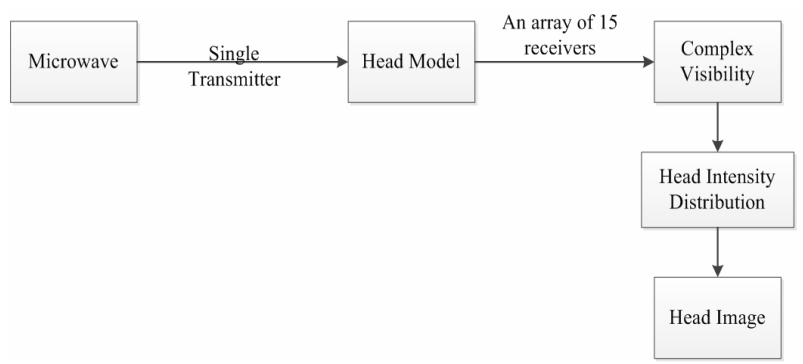

Figure 1. Block diagram of HMIA system.

Figure 2 shows the 3D geometry relevant to HMIA system. If two points $P(x, y, z)$ and $P^{\prime}\left(x^{\prime}, y^{\prime}, z^{\prime}\right)$ are assumed within the head, the visibility function of the backscattered electric field $\underline{E}_{\text {scat }}$ within the head for any two antennas $A_{i}$ and $A_{j}$ located at $r_{i}$ and $r_{j}$ is defined:

$$
G\left(\underline{r_{i}}, \underline{r_{j}}\right)=<E_{\text {scat }}\left(\underline{r_{i}}\right) \cdot E_{\text {scat }}^{*}\left(r_{\underline{j}}\right)>
$$

Where $*$ denotes the complex conjugate and $<>$ stands for the expected value (time average).

It is well-known that the scattered electric field can be represented as an integral over the volume of the scatterer involving the induced polarisation currents that arise from the complex permittivity contrast with the host medium [12]. In the far-field of the antenna, the scattered field can then be written as follows:

$$
\underline{E}_{\text {scat }}(\underline{r})=\left(\frac{k_{0}^{2}}{4 \pi}\right) \int_{V}\left(\varepsilon(\underline{s})-\varepsilon_{b}\right) \underline{\mathrm{E}}_{\mathrm{tot}}(\underline{\mathrm{s}}) \frac{\mathrm{e}^{-\mathrm{j} \mathrm{k}_{\mathrm{b}}|\underline{\mathrm{s}}-\underline{\mathrm{r}}|}}{|\underline{\mathrm{s}}-\underline{\mathrm{r}}|} \mathrm{dV}
$$

Where in (2) $\underline{E}_{t o t}(\underline{s})=$ Total electric field (incident plus scattered) at a point inside the head with position
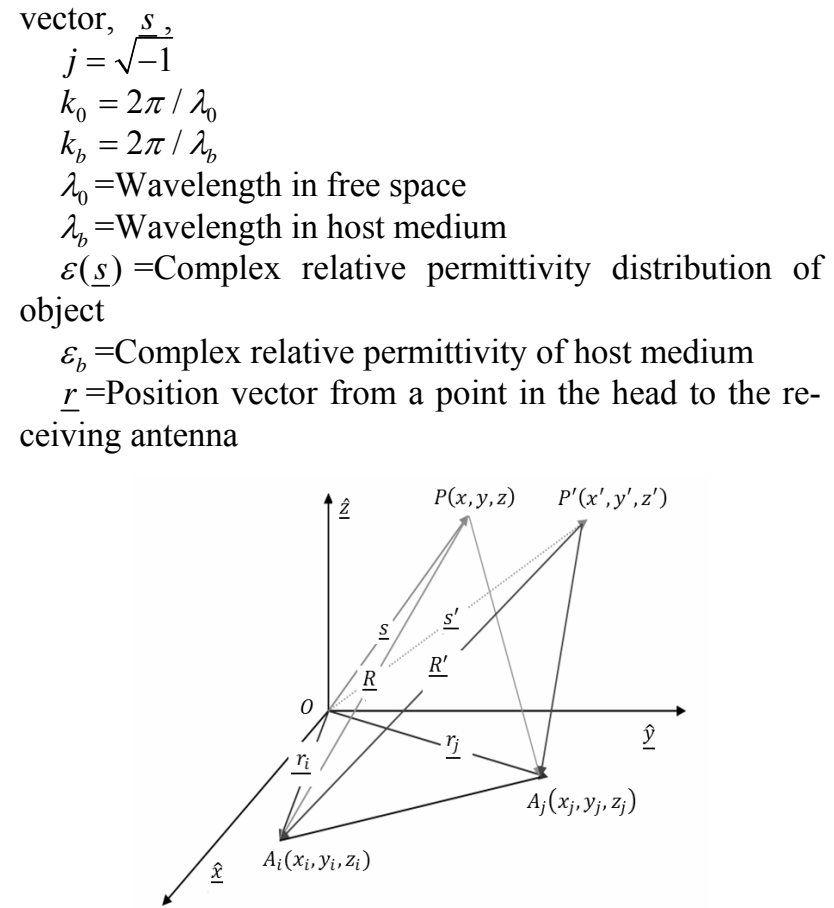

Figure 2. 3D geometry of HMIA measurement.

Substituting for the scattered fields in (1) using (2) gives the following six-fold integral for the complex visibility function:

$$
\begin{aligned}
& \mathrm{G}\left(\underline{\mathrm{r}}_{i}, \mathrm{r}_{j}\right) \\
& =\left(\frac{\mathrm{k}_{0}^{2}}{4 \pi}\right)^{2} \iiint \iiint_{\mathrm{V}} \int_{\mathrm{V}^{\prime}}\left(\varepsilon(\overline{\mathrm{s}})-\varepsilon_{b}\right)\left(\varepsilon\left(\underline{\mathrm{s}^{\prime}}\right)-\varepsilon_{b}\right)^{*} \underline{\mathrm{E}}_{\mathrm{tot}(\underline{\mathrm{s}})} \\
& \cdot \underline{\mathrm{E}}_{\mathrm{tot}}^{*}\left(\underline{\mathrm{s}^{\prime}}\right) \frac{\mathrm{e}^{-\mathrm{jk} \mathrm{R}_{b}\left(\mathrm{R}-\mathrm{R}^{\prime}\right)}}{\mathrm{RR}^{\prime}} \mathrm{dVdV} \mathrm{V}^{\prime}
\end{aligned}
$$

Where in (3) $R=\left|\underline{r_{i}}-\underline{s}\right|$

$$
R^{\prime}=\left|\underline{r_{j}}-\underline{s}\right|
$$

If the distance from a point $P$ the receiving antenna $A_{i}$ is very large compared to the size of antenna array plane, that is, $R \gg\left|r_{i}\right|$, then:

$$
\begin{aligned}
& \mathrm{R}=|\underline{\mathrm{R}}|=\sqrt{\left(\underline{\mathrm{r}_{i}}-\underline{\mathrm{s}}\right) \cdot\left(\underline{\left.r_{i}-\underline{\mathrm{s}}\right)}\right.}=\sqrt{\mathrm{r}_{i}^{2}+\mathrm{s}^{2}-2 \underline{\mathrm{r}_{i}} \cdot \underline{\mathrm{s}}} \\
& \cong \mathrm{s}-\frac{\mathrm{r}_{i} \cdot \underline{\mathrm{s}}}{\mathrm{s}}=\mathrm{s}-\underline{\mathrm{r}_{i}} \cdot \underline{\hat{\mathrm{s}}}
\end{aligned}
$$

Where the "dot" denotes scalar product and $\underline{\hat{s}}$ is a unit vector. Similarly, the distance from another point $P^{\prime}$ in the head to the receiving antenna $A_{j}$ can be calculated as:

$$
\mathrm{R}^{\prime}=\left|\underline{\mathrm{R}^{\prime}}\right|=\mathrm{s}^{\prime}-\underline{\mathrm{r}_{j}} \cdot \underline{\mathrm{s}^{\prime}}
$$

Then

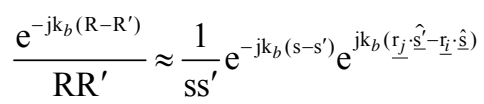


The six-fold integral of (3) can be simplified by noting that the phase factor $e^{-j k_{b}\left(s-s^{\prime}\right)}$ oscillates rapidly as we scan over all possible pairs of points $\left(P, P^{\prime}\right)$ within the domain of integration. Consequently, the only significant contribution to the value of the integral in (3) arises from points for which the phase varies slowly. This situation corresponds to the case for which the points $\left(P, P^{\prime}\right)$ coincide. Therefore, we allow $\underline{s}-\underline{s^{\prime}} \rightarrow 0$ so that $s=s^{\prime}$, and obtain the following volume integral for the visibility function where the integration is over the volume of the scatterer, $V$ :

$$
\begin{aligned}
& \mathrm{G}\left(\underline{\mathrm{r}}_{\underline{\underline{\mathrm{r}}}}, \mathrm{r}_{\underline{j}}\right) \\
& =\left(\frac{\mathrm{k}_{0}^{2}}{4 \pi}\right)^{2} \iiint_{\mathrm{V}}\left(\left|\varepsilon(\underline{\mathrm{s}})-\varepsilon_{b}\right|^{2}\right) \underline{\mathrm{E}}_{\mathrm{tot}(\overline{\mathrm{s}})} \\
& \cdot \underline{\mathrm{E}}_{\mathrm{tot}}^{*}\left(\underline{\mathrm{s}}^{\prime}\right) \frac{\mathrm{e}^{-\mathrm{j} \mathrm{k}_{b}\left(\underline{\mathrm{r}}_{\mathrm{i}}-\mathrm{r}^{\prime}\right)} \cdot \hat{\underline{\underline{s}}}}{\mathrm{~s}^{2}} \mathrm{dV}
\end{aligned}
$$

Defining the head intensity function at the position $\underline{s}$ as:

$$
I(\underline{s})=\left(\frac{k_{0}^{2}}{4 \pi}\right)^{2}\left|\varepsilon(\underline{s})-\varepsilon_{b}\right|^{2} \underline{E}_{t o t}(\underline{s}) \cdot \underline{E}_{t o t}^{*}(\underline{s})
$$

Equation (7) can be written as:

$$
G(\underline{D})=\iiint_{V} I(\underline{s}) \frac{e^{-j 2 \pi \underline{D} \cdot \hat{s}}}{s^{2}} d V
$$

In (9), $\underline{D}=\left(\underline{r_{i}}-\underline{r_{j}}\right) / \lambda_{b}$

A more useful visibility form obtained by using spherical polar coordinate system as shown in Figure 3:

$$
G(\underline{D})=\iiint_{V} I(\underline{s}) \frac{e^{-j 2 \pi \underline{D} \cdot \hat{s}}}{n} d l d m d s
$$

Where $\quad \underline{\hat{s}}=\sin \theta \cos \phi \underline{\hat{x}}+\sin \theta \sin \phi \hat{y}+\cos \theta \underline{\hat{z}}$ $l=\sin \theta \cos \phi, \quad m=\sin \theta \sin \phi, \quad n=\cos \theta=\sqrt{1-l^{2}-m^{2}}$.

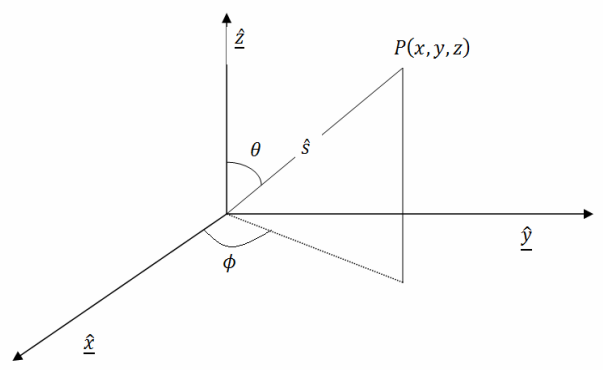

Figure 3. Spherical polar coordinate system.

Writing the Cartesian components of the baseline vector $\underline{D}$ as $(u, v, w)$ such that:

$$
\begin{aligned}
& u=\left(x_{2}-x_{1}\right) / \lambda_{\mathrm{b}} \\
& v=\left(y_{2}-y_{1}\right) / \lambda_{\mathrm{b}} \\
& w=\left(z_{2}-z_{1}\right) / \lambda_{\mathrm{b}}
\end{aligned}
$$

the visibility function then becomes:

$$
\begin{aligned}
& G(u, v, w) \\
& =\iiint_{l m s} \frac{I(s, l, m)}{\sqrt{1-l^{2}-m^{2}}} e^{-j 2 \pi \Phi} d l d m d s
\end{aligned}
$$

Where $\Phi=\underline{D} \cdot \underline{\hat{s}}=u l+v m+w n$

If all antennas are assumed to be located on a 2D plane then it follows that $w=0$. We now define a line integral along the radial coordinate, $s$, so that:

$$
\tilde{I}(l, m)=\int_{s} \frac{I(s, l, m)}{\sqrt{1-l^{2}-m^{2}}} d s
$$

Using (13) leads to the following 2D integral over the variables $(l, m)$ for the visibility function:

$$
G(u, v, 0)=\iint \tilde{I}(l, m) e^{-j 2 \pi(u l+v m)} d l d m
$$

It is evident that the visibility function in (14) is the 2D Fourier transform of the 2D intensity function $\tilde{I}(l, m)$ which is consistent with the Van Cittert-Zernike theorem [13]. Therefore, by inverse Fourier we obtain for the $2 \mathrm{D}$ intensity function:

$$
\tilde{I}(l, m)=\iint G(u, v, 0) e^{j 2 \pi(u l+v m)} d u d v
$$

The $2 \mathrm{D}$ image is the intensity function $\tilde{I}(l, m)$ which is defined by the line integral in (13) and represents the scattering intensity in the head integrated along each radial vector.

\section{Simulation}

\subsection{Antenna and Scattered Field Model}

A small open-ended rectangular waveguide antenna was simulated as the transmitter and receiver.The typical dimensions of the antenna are $10 \mathrm{~mm}$ and $7 \mathrm{~mm}$. The incident field of such antenna is given by:

$$
\begin{aligned}
& \underline{E}_{\text {inc }}\left(R_{0}, \theta, \phi\right) \\
& =\frac{-j k_{b}}{2 \pi^{2}} \underline{E}_{0} \frac{e^{-j k_{b} R_{0}}}{R_{0}} A B h(\theta, \phi) \underline{P}(\theta, \phi)
\end{aligned}
$$

Where $\underline{E}_{0}=$ Wave amplitude of $T E_{0}$ model within waveguide aperture

$R_{0}=$ Distance from a point in the head to the transmitting antenna

$A=$ Broad aperture dimension of antenna aperture

$B=$ Narrow aperture dimension of antenna aperture

$h(\theta, \phi)=$ Antenna far-field radiation pattern

$\underline{P}(\theta, \phi)=$ Polarisation vector

The backscattered electric field from the head can be found by applying the Born approximation[10] which gives the following integral over the volume of the head:

$$
\begin{gathered}
\bar{E}_{\text {scat }}=\frac{k_{0}^{2}}{4 \pi} \int_{V}\left(\varepsilon(\bar{s})-\varepsilon_{b}\right)\left[\bar{E}_{i n c}-\left(\bar{E}_{i n c}\right.\right. \\
\cdot \underline{\hat{R}}) \underline{\hat{R}}] \frac{e^{-j k_{b} R}}{R} d V
\end{gathered}
$$

A computer simulation model was developed using 
MATLAB by combining (17) and (1) to simulate the complex visibility function that is detailed in section 2 . The head intensity distribution function $\tilde{I}$ was used to generate a $2 \mathrm{D}$ head image.

\subsection{Antenna Array}

An array of 16 antennas including one transmitter and 15 receivers (Figure 4.) was placed under the head model in far-field distance $(\mathrm{z}=-450 \mathrm{~mm})$.A matching solution medium was used in the space between antenna array and the head model.

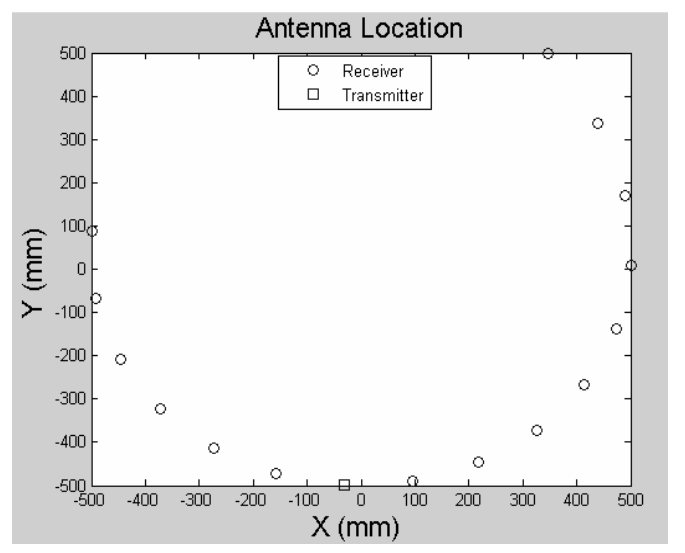

Figure 4. Schematic of array configuration.

\subsection{Head Model}

Figure 5 displays the 2D and 3D views of a 3D head model that contains skin, fat, skull, cerebral spin fluid (CSF), grey matter, white matter and an ischemic or hemorrhagic stroke area. The ellipse-shaped head model has major radius of $100 \mathrm{~mm}$, minor radius of $85 \mathrm{~mm}$, and height of $100 \mathrm{~mm}$. The dielectric properties of the head model are summarized in Table 1[5-6].

\section{Simulation Results}

The $100 \mathrm{~mm} \times 100 \mathrm{~mm}$ square region containing the object (head) and the background medium (matching solution with the relative permittivity of $\varepsilon_{r}=40-13 \mathrm{j}$ ) is uniformly subdivided into $401 \times 401$ elementary square cells. Figure 6 shows 2D view of the original and reconstructed head images without stroke. Color bar in Figure 6 (a) plots the dielectric properties of head model, and colour bar in Figure 6 (b) plots signal energy on a linear scale, normalized to the maximum in the $2 \mathrm{D}$ head area.

Figure 7(a) shows the original head image contains a small ischemic stroke $(5 \mathrm{~mm}$ diameter spherical ball, located at $X=-40, Y=0, Z=-25$ ). Figure 7 (b) clearly shows the simulated ischemic within the reconstructed 2D head image.

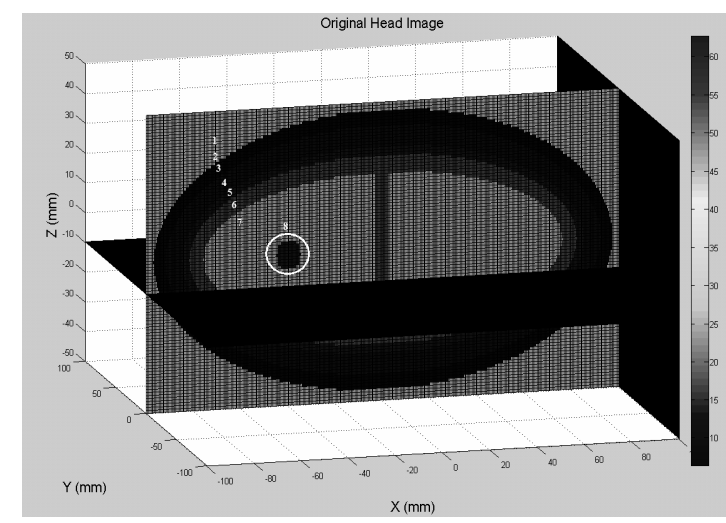

(a)

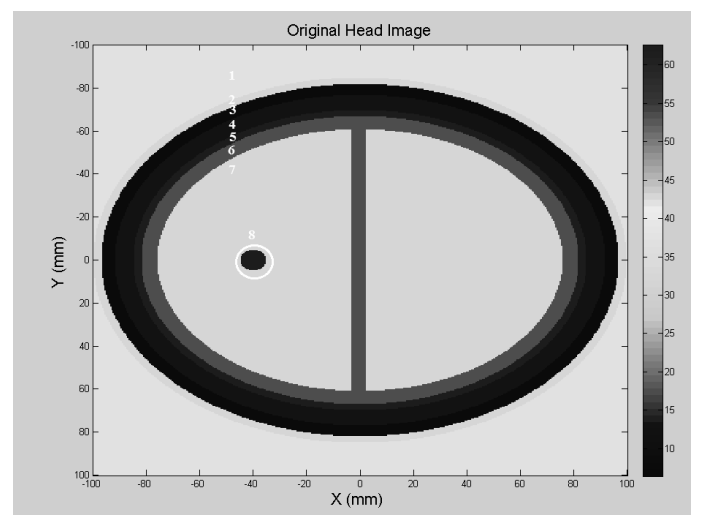

(b)

Figure 5. The simulated 3D head model with a stroke (5 $\mathrm{mm}$ diameter spherical ball) located at $(X=0 \mathrm{~mm}, \mathrm{Y}=\mathbf{- 4 0}$ $\mathrm{mm}, \mathrm{Z}=0 \mathrm{~mm}$ ) (a) $3 \mathrm{D}$ view (b)2D view (1:Matching solution, 2: Skin, 3: Fat, 4: Skull, 5: CSF, 6: Grey matter, 7: White matter, 8: Ischemic/Hemorrhagic stroke).

Table 1. Dielectric properties of head at $2.5 \mathrm{GHz}$ [5-6].

\begin{tabular}{|c|c|c|c|}
\hline No & Region & $\begin{array}{c}\text { Thickness } \\
(\mathrm{mm})\end{array}$ & $\begin{array}{c}\text { Dielectric } \\
\text { properties }\end{array}$ \\
\hline 1 & Matching Solution & & $40-13 \mathrm{j}$ \\
\hline 2 & Skin & 3 & $41-11 \mathrm{j}$ \\
\hline 3 & Fat & 5 & $5-4 \mathrm{j}$ \\
\hline 4 & Skull & 7 & $13-2 \mathrm{j}$ \\
\hline 5 & CSF & 3 & $57-26 \mathrm{j}$ \\
\hline 6 & Grey matter & 6 & $50-18 \mathrm{j}$ \\
\hline 7 & White matter & & $40-15 \mathrm{j}$ \\
\hline 8 & Ischemic Stroke & 5 & $36-13 \mathrm{j}$ \\
\hline 8 & $\begin{array}{c}\text { Hemorrhagic } \\
\text { Stroke }\end{array}$ & 10 & $61-13 \mathrm{j}$ \\
\hline
\end{tabular}




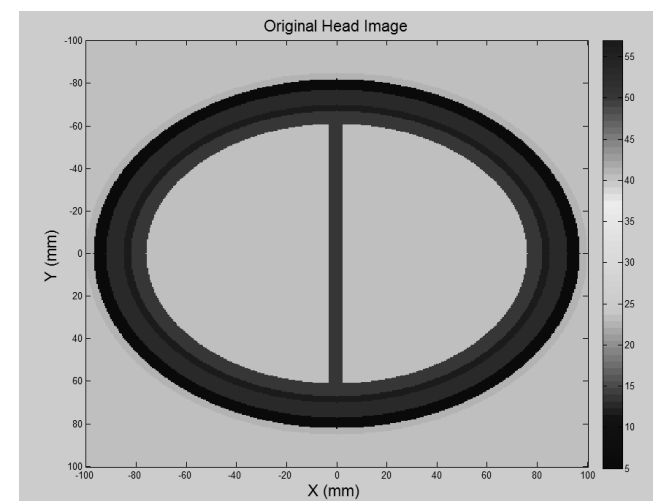

(a)

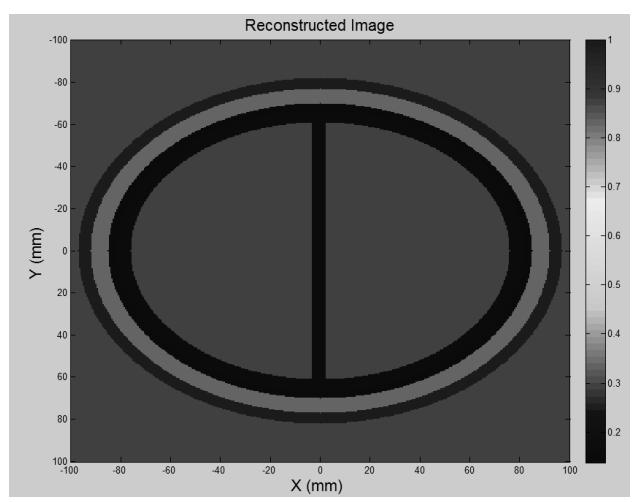

(b)

Figure 6. (a) Original head model without stroke (b) Reconstructed image of simulated head model.

Figure 8 (a) shows the original head image contains a hemorrhagic stroke $(10 \mathrm{~mm}$ diameter spherical ball, located at $X=40, Y=0, Z=25$ ). Figure 8 (b) clearly show the simulated hemorrhagic stroke within the reconstructed 2D head image.

Colour bars in Figure 7 (a) and Figure 8 (a) plot the dielectric properties of head model, and colour bars in Figure 7 (b) and Figure 8 (b) plot signal energy on a linear scale, normalized to the maximum in the $3 \mathrm{D}$ head volume.

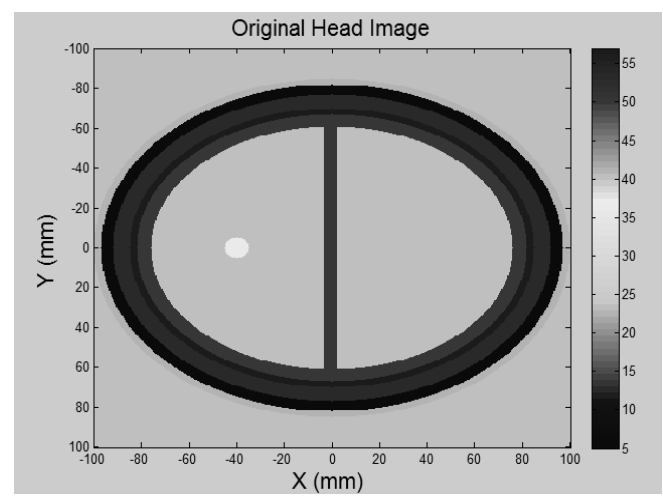

(a)

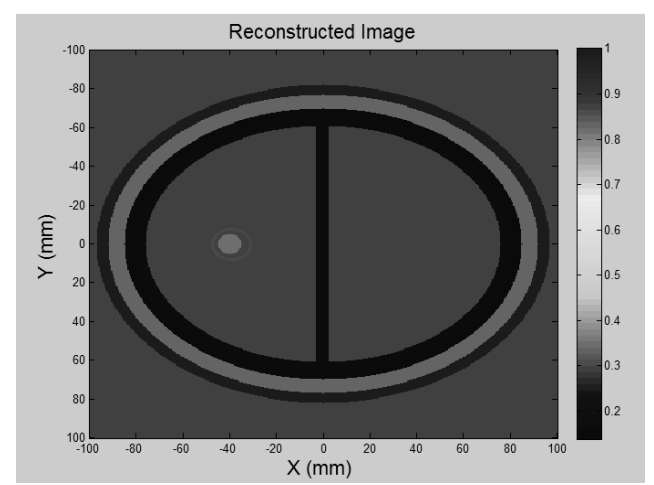

(b)

Figure 7. (a) Original head model contains one ischemic stroke (b)Reconstructed image of simulated head model.

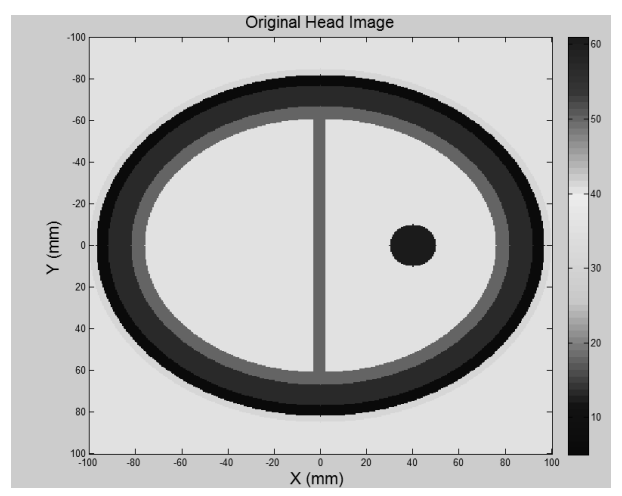

(a)

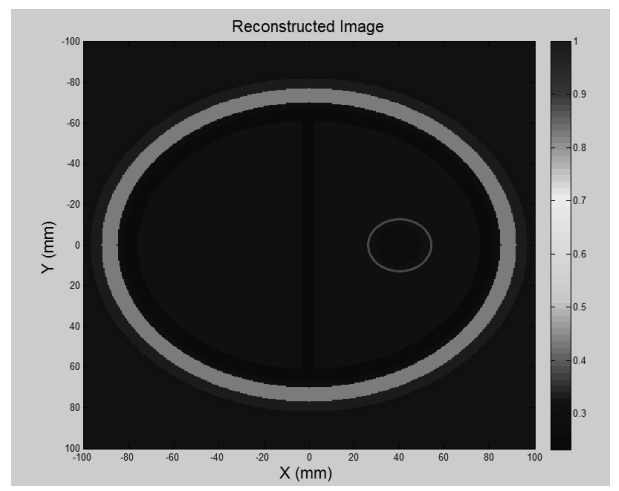

(b)

Figure 8. (a) Original head model contains one hemorrhagic stroke (b)Reconstructed image of simulated head model.

\section{Conclusions}

This simulation paper has described a new image reconstruction algorithm for head imaging and brain stroke detection. The algorithm was developed based on the holographic microwave imaging technique with using a single frequency of $2.5 \mathrm{GHz}$.

A computer simulation model was developed using 
MATLAB to demonstrate that the HMIA can produce good quality head images.

Simulation results demonstrated that is chemic and hemorrhagic inside of a high dielectric contrast shield, comprising the skull and cerebral spinal fluid could be detected. The HMIA technique has potential benefits such as significant improvement of imaging results compare to other microwave imaging approaches, simplicity, safety and comfort compared to other screening methods, such as CT scanning.

\section{Acknowledgements}

The authors gratefully acknowledge the support of the Institute of Biomedical Technologies (IBTec) at the Auckland University of Technology (AUT), and the support of Callaghan Innovation, Auckland, New Zealand.

\section{REFERENCES}

[1] A. S. Mozaffarian, D. Roger, V. L. Benjamin, E. J. Berry, J. D. Borden and M. B. Turner, "Heart Disease and Stroke Statistics - 2013 Update A Report From the American Heart Association," Circulation, Vol. 127, No. 1, 2013. doi:10.1161/CIR.0b013e31828124ad

[2] B. J. Mohammed, A. M. Abbosh, D. Ireland and M. E. Bialkowski, "Compact Wideband Antenna for Microwave Imaging of Brain," Progress In Electromagnetics Research C, Vol. 27, 2012, pp. 27-39. doi:10.2528/PIERC11102708

[3] K. W. Muir, A. Buchan, R. Von Kummer, J. Rother and J. C. Baron, "Imaging of Acute Stroke," The Lancet Neurology, Vol. 5, No. 9, 2006, pp. 755-768. doi:10.1016/S1474-4422(06)70545-2

[4] R. Scapaticci, L. Di Donato, I. Catapano and L. Crocco, "A Feasibility Study on Microwave Imaging for Brain
Stroke Monitoring," Progress In Electromagnetics Research B, Vol. 40, 2012, pp. 305-324.

[5] A. Peyman, S. J. Holden, S. Watts, R. Perrott and C. Gabriel, "Dielectric Properties of Porcine Cerebrospinal Tissues at Microwave Frequencies: In Vivo, in Vitro and Systematic Variation with Age," Physics in medicine and biology, Vol. 52, No. 8, 2007, p. 2229. doi:10.1088/0031-9155/52/8/013

[6] S. Gabriel, R. W. Lau and C. Gabriel, "The Dielectric Properties of Biological Tissues: II. Measurements in the Frequency Range $10 \mathrm{~Hz}$ to $20 \mathrm{GHz}$," Physics in medicine and biology, Vol. 41, No. 11, 1996, pp.2251. doi:10.1088/0031-9155/41/11/002

[7] S. Y. Semenov and D. R. Corfield, "Microwave TomoGraphy for Brain Imaging: Feasibility Assessment for Stroke Detection," International Journal of Antennas and Propagation, 2008. doi:10.1155/2008/254830

[8] D. Ireland and M. Bialkowski, "Feasibility Study on Microwave Stroke Detection Using a Realistic Phantom and the FDTD Method," Microwave Conference Proceedings (APMC), 2010 Asia-Pacific, 2010, pp. 1360-1363.

[9] H. Trefna and M. Persson, "Antenna Array Design for Brain Monitoring," Antennas and Propagation Society International Symposium, 2008, pp.1-4.

[10] L. Wang, R. Simpkin, and A. M. Al-Jumaily, "Holography Microwave Imaging Array for Early Breast Cancer Detection,"Proceedings of 2012 ASME International Mechanical Engineering Congress \& Exposition, Houston, Texas, United States, 2012.

[11] L. Wang, R. Simpkin and A. M. Al-Jumaily, "3D Breast Cancer Imaging Using Holographic Microwave InterFerometry," Proceedings of the 27th Conference on Image and Vision Computing New Zealand, pp. 180-185.

[12] S. Silver, "Microwave Antenna Theory and Design, MIT Radiation Laboratory Series," Vol. 10, 1949, p. 87.

[13] M. Born and E. Wolf, "Principles of Optics: ElectroMagnetic Theory of Propagation, Interference and Diffraction of Light," Pergamon Press, Sixth Edition, 1980, p. 510. 